\title{
Retraction Note: Unexpected events disrupt visuomotor working memory and increase guessing
}

\author{
R. Dawn Finzi ${ }^{1} \cdot$ Bradley R. Postle $^{2} \cdot$ Timothy F. Brady $^{1} \cdot$ Adam R. Aron $^{1}$
}

Published online: 17 July 2018

(C) Psychonomic Society, Inc. 2018

\section{Retraction Note: Psychon Bull Rev https://doi.org/10.3758/s13423-017-1319-3}

The authors are retracting this article (Finzi et al., 2018) because after publication they discovered a mistake in the behavioral analysis. This affects the results shown in Fig. 2 and Table 1 . The mistake is that, at the single-subject level, the mean response error variable was calculated for novels and standards after removing outliers separately for these two conditions. However, because there were different numbers of novel and standard trials, this method inflated the mean difference between conditions. Specifically, it produced a bias toward increased response errors on novels versus standards, as can be seen in Fig. 2, which we confirmed with simulations. When outliers were removed in an appropriate fashion, the difference between means across all three experiments was much reduced and not significant. However, a single-subject analysis clearly showed that novel trials have an increased proportion of trials with large response errors (i.e., there is a mixture of two distributions), as compared to standards. The mixture modeling approach of Experiment 3 captured this, and the increased guessing rate for novels versus standards remains significant there, as reported in the article. Unfortunately, the authors cannot fit that mixture model to the data from Experiments 1 and 2, because the position of stimuli on the screen was not set up in those experiments to be equidistant (as it was in Exp. 3). In summary, the authors have confidence in a core result from the article: that novel events increase guessing when they are presented in the delay period of a visuomotor task. However, the effect is more subtle that they thought, and because Fig. 2 and Table 1 have incorrect values, they are retracting this article. The authors sincerely regret any inconvenience to readers. All authors agree with this retraction.

The online version of the original article can be found at https://doi.org/ $10.3758 / \mathrm{s} 13423-017-1319-3$

Adam R. Aron

adamaron@ucsd.edu

1 Psychology Department, University of California, San Diego, La Jolla, USA

2 Psychology Department, University of Wisconsin-Madison, Madison, USA 